(2) Open Access Full Text Article

\title{
Lower conjunctival fornix packing for mydriasis in premature infants: a randomized trial
}

\author{
Onsiri Thanathanee' \\ Tanapat Ratanapakorn' \\ Michael G Morley² \\ Yosanan Yospaiboon' \\ 'Department of Ophthalmology, \\ Faculty of Medicine, Khon Kaen \\ University, Khon Kaen, Thailand; \\ ${ }^{2}$ Harvard Medical School, \\ Boston, MA, USA
}

This article was published in the following Dove Press journal:

Clinical Ophthalmology

14 February 2012

Number of times this article has been viewed
Objective: To compare the mydriatic effect of lower conjunctival fornix packing to conventional instillation of eyedrops containing $2.5 \%$ phenylephrine and $1 \%$ tropicamide in premature infants undergoing examination for retinopathy of prematurity.

Methods: The patients were randomized to receive either conventional instillation of mydriatic drops or lower conjunctival fornix packing in one eye and the alternate method in the fellow eye. For the eyes receiving lower conjunctival fornix packing (study group), one small piece of the cotton wool soaked with one drop of $2.5 \%$ phenylephrine and one drop of $1 \%$ tropicamide was packed in the lower conjunctival fornix for 15 minutes. For the eyes receiving the conventional instillation (control group), $2.5 \%$ phenylephrine and $1 \%$ tropicamide were alternately instilled every 5 minutes for two doses each. Horizontal pupil diameter was measured with a ruler in millimeters 40 minutes later.

Results: The mean dilated pupil diameter in study group and control group were $5.76 \pm 1.01 \mathrm{~mm}$ and $4.50 \pm 1.08 \mathrm{~mm}$, respectively. This difference was statistically significant $(P<0.05)$.

Conclusion: The dilated pupil diameter after receiving the lower conjunctival fornix packing was larger than conventional instillation with a statistically significant difference. We recommended the packing method to dilate the preterm infant pupil, especially if the pupil is difficult to dilate.

Keywords: conjunctival fornix packing, phenylephrine, mydriasis, premature infants

\section{Introduction}

Adequate pupil dilation is needed for the screening and treatment of retinopathy of prematurity (ROP) in premature infants. ${ }^{1}$ In clinical practice, mydriatic drugs are instilled several times until the pupil reaches maximum size. However, mydriasis may be suboptimal in many eyes, despite maximum instillation.

From clinical observation, we found that packing of mydriatic drugs in the lower conjunctival fornix was more effective in producing pupil dilation than topical instillation of eyedrops. The purpose of this study was to compare the mydriatic effect of $2.5 \%$ phenylephrine and $1 \%$ tropicamide when delivered by lower conjunctival fornix packing with topical instillation of these eyedrops in premature infants.

\section{Materials and methods}

This study was conducted according to the principles expressed in the Declaration of Helsinki. It was reviewed and approved by the Khon Kaen University ethics committee for human research. The sample size was calculated from the formula: $s^{2}\left(Z_{\alpha}+Z_{\beta}\right)^{2} / \Delta^{2}$. The study was powered to detect a difference of $0.5 \mathrm{~mm}$ with a certainty of $95 \%$, 
and the standard deviation of 0.9 from the previous study was used. ${ }^{2}$ The required number of patients per treatment group was 25. From May 2007 to July 2007, 25 preterm infants of Thai ethnicity in the newborn unit at Srinagarind Hospital, Khon Kaen University were enrolled in this study. The informed consent forms were signed by their parents in all cases. The inclusion criteria consisted of premature infants who had a gestational age less than 30 weeks, a birth weight less than $1500 \mathrm{~g}$, or a birth weight between 1500 and $2000 \mathrm{~g}$ with an unstable clinical course. Exclusion criteria included those with history of intraocular surgery or laser treatment, previous eye drop instillation that might affect the pupil size, severe underlying disease, or unstable clinical course in which mydriatic drugs or the examination could aggravate their conditions.

The patients were randomized to either conventional instillation or lower conjunctival fornix packing in one eye and the other method in the other eye. The randomization allocation sequence was generated using a computer-generated random numbers table (SAS software, SAS Institute, Inc, Cary, NC). The randomization allocation was concealed by using sealed opaque envelopes numbered sequentially using the sequence described above, and kept by a research assistant. The research assistant assigned the patients to their groups according to the random allocation sequence. Both the patients and assessors were blinded to group assignment and unblinding was done at the end of the study. For the eyes receiving lower conjunctival fornix packing (study group), one small piece of the sterile cotton wool soaked with one drop of $2.5 \%$ phenylephrine and one drop of $1 \%$ tropicamide was packed in the lower conjunctival fornix for 15 minutes. For the eyes receiving the topical instillation (control group), 2.5\% phenylephrine and $1 \%$ tropicamide were alternately instilled every 5 minutes for two doses each. The main outcome measurement was the horizontal pupil diameter measured with a ruler in $0.5 \mathrm{~mm}$ increments 40 minutes later. We measured three times to improve the reliability. Systolic blood pressure, diastolic blood pressure, and heart rate were measured immediately prior to pupil dilation and 40 minutes after when the infants were quiet. The data were analyzed using a paired $t$-test to compare the mean pupil diameter, systolic blood pressure, diastolic blood pressure, and heart rate of both groups. Statistical significance was taken as $P<0.05$.

\section{Results}

The demographic characteristics of the patients including gestational age, age at the time of study, birth weight, and weight at the time of study are shown in Table 1 . The mean
Table I Demographic characteristics of the patients

\begin{tabular}{llc}
\hline Variables & Range & Mean \pm SD \\
\hline GA (at birth) & $24-36$ weeks & $30.7 \pm 3.6$ weeks \\
GA (at exam) & $28-43$ weeks & $35.8 \pm 3.3$ weeks \\
BW (at birth) & $375-2180 \mathrm{~g}$ & $1317.2 \pm 419.6 \mathrm{~g}$ \\
BW (at exam) & I I40-4000 g & $1976.3 \pm 701.1 \mathrm{~g}$ \\
\hline
\end{tabular}

Abbreviations: GA, gestational age; BW, body weight; SD, standard deviation.

pupil diameter at baseline and after mydriatric drugs (both topical and fornix packing) are shown in Table 2. The mean pupil diameter at baseline was equal in both groups. The fornix packing group pupil size was larger than the topical instillation group with a statistically significant difference (mean difference $1.26 \mathrm{~mm}, 95 \%$ confident interval [CI]: $0.81-1.71, P<0.05)$. In the 25 patients studied, there was one case in which the pupil size of the fornix packing eye $(5 \mathrm{~mm})$ was smaller than the eye which received topical instillation (6 mm). In this case, the pledget was not placed deeply enough in the fornix and it slipped out; however, data was still collected. In another case the pupil size in both groups was equal (4 mm), with no deviations from protocol. In the remaining 23 patients, the fornix packing eye had a measurably larger pupil. The mean systolic blood pressure, diastolic blood pressure, and heart rate before and after the procedure are shown in Table 3. There was no statistically significant difference in mean systolic blood pressure. However, there were statistically significant differences in mean diastolic blood pressure and heart rate.

\section{Discussion}

In our study, we observed that the lower conjunctival fornix packing of the mydriatric drugs was better than the conventional topical instillation method in several respects. It yields a larger pupil size for complete peripheral fundus examination. The measured side effects showed a mild increase in heart

Table 2 Comparison of mean $( \pm S D)$ in the pupil size between two groups

\begin{tabular}{lll}
\hline Variables & Range $(\mathbf{m m})$ & Mean \pm SD $(\mathbf{m m})$ \\
\hline Before mydriasis & & \\
$\quad$ Pupil size (RE) & $2-3$ & $2.2 \pm 0.3$ \\
$\quad$ Pupil size (LE) & $2-3$ & $2.2 \pm 0.3$ \\
After mydriasis & & \\
$\quad$ Pupil size (packing) & $4-8$ & $5.8 \pm 1.0$ \\
$\quad$ Pupil size (instillation) & $3-6$ & $4.5 \pm 1.1$ \\
Mean difference & $1.3 \pm \mathrm{I} . \mathrm{I}(95 \% \mathrm{Cl}:$ & \\
& $0.8-\mathrm{I} .7)$ & \\
\hline
\end{tabular}

Abbreviations: SD, standard deviation; RE, right eye; $\mathrm{LE}$, left eye; $\mathrm{Cl}$, confidence interval. 
Table 3 Mean systolic blood pressure, diastolic blood pressure, and heart rate of the patients

\begin{tabular}{lccl}
\hline & Before packing & After packing & $P$ value \\
\hline Blood pressure $(\mathrm{mmHg})$ & & & \\
$\quad$ Systolic & $62.5 \pm 6.9$ & $64.4 \pm 6.6$ & 0.12 \\
Diastolic & $34.4 \pm 3.5$ & $42.2 \pm 4.1$ & $<0.01$ \\
Heart rate $(\mathrm{bpm})$ & $143.5 \pm 13.0$ & $148.5 \pm 12.6$ & $<0.01$ \\
\hline
\end{tabular}

Abbreviation: bpm, beats per minute.

rate and diastolic blood pressure, but the net effect is no apparent increase in real danger or risk to the baby. Also, the pledget may result in more drugs getting into the eye and body even though the amount is only one drop, due to the reservoir function of the pledget. Furthermore, we could also save time spent on instillation. This study confirmed that the mean pupil diameter of the packing group was larger than the instillation group with a statistically significant difference, which supported our hypothesis.

Sindel et $\mathrm{al}^{3}$ compared the pupillary and cardiovascular effects of various mydriatic agents in preterm infants and found that the mean pupil size following the instillation method was larger than those in our study. This may be attributed to the different measuring times. They measured the pupil diameter at 60 minutes after the administration compared to 40 minutes in our study. Other possible causes may be due to racial factors such as iris pigmentation. Howard and $\mathrm{Lee}^{4}$ first demonstrated that lightly pigmented irides responded to smaller doses of mydriatic drugs and yielded a larger mydriasis, which developed more rapidly. Yospaiboon et $\mathrm{al}^{5}$ also demonstrated that in darkly pigmented irides, $10 \%$ phenylephrine was significantly more effective than $2.5 \%$ phenylephrine in pupil dilation, whereas there was no significant difference between both groups in Caucasian studies. Angenent and Koelle ${ }^{6}$ postulated that the difference might be due to increased destruction of the sympathetic transmitter in pigmented irides. From an experimental study in rabbits, they found that adrenaline was oxidized more rapidly by homogenates of pigmented irides than of albino irides. This was attributed to the presence of a more active catechol-oxidase system. Emiru ${ }^{7}$ postulated that phenylephrine had to be absorbed through the cornea into the aqueous humor and then absorbed by the iris surface. In darkly pigmented irides, the anterior layer of the iris was thicker due to denser iris chromatophores and had fewer crypts. This made phenylephrine absorption much slower than in lightly pigmented irides which had a thinner anterior layer and more numerous and larger crypts.

Khoo et $\mathrm{al}^{8}$ studied the mydriatric effect of $2.5 \%$ phenylephrine and $0.5 \%$ tropicamide in preterm infants with dark irides and found that the mean pupil diameter was smaller than those in light pigmented irides studies, but larger than our result. This may be attributed to more doses and measuring time at 60 minutes after instillation. Chew et $\mathrm{al}^{9}$ also conducted a cross-sectional, randomized, double-masked clinical trial comparing cyclopentolate $1 \%$ and phenylephrine $2.5 \%$, tropicamide $1 \%$, and phenylephrine $2.5 \%$, and a prepared combination of cyclopentolate $0.2 \%$ with phenylephrine $1 \%$ for pupil dilation in preterm infants with dark irides. They concluded that the prepared combination of cyclopentolate $0.2 \%$ with phenylephrine $1 \%$ was the mydriatic of choice for preterm infants with dark irides as it provided adequate pupil dilation with the least systemic side effects.

There were only a few studies comparing packing with the instillation method to deliver preoperative mydriatics for cataract surgery in adults. Ong-Tone ${ }^{10}$ used a wick soaked in dilating mixture containing cyclopentolate $1 \%$, phenylephrine $2.5 \%$, diclofenac sodium $0.1 \%$, and ofloxacin $0.3 \%$ and packed in lower conjunctival fornix compared with use of eyedrops. They found that the difference in pupil diameter between two groups was not statistically significant, but the time was saved. McCormick et $\mathrm{al}^{11}$ also found that the use of a pledget cellulose sponge to deliver mydriatics prior to cataract surgery is as effective as the conventional method of repeated drop administration and was not associated with any adverse effects. Dubois et $\mathrm{al}^{12}$ conducted a prospective randomized masked trial comparing the effect of mydriatic drops (tropicamide 1\%, phenylephrine $2.5 \%$, diclofenac sodium $0.1 \%$ ) instilled prior to surgery, and a wick soaked in the same drop mixture placed in the inferior fornix. The result also showed no significant difference between the mydriasis obtained with the depot system compared with the conventional drop application.

Our study demonstrated that the packing method was more effective than instillation with a statistically significant difference. This may be explained by racial factors as mentioned before. Another factor that may explain this result was the size of the conjunctival fornix. Neonates have a much smaller area of conjunctival fornix than adults so mydriatric drugs are less absorbed via the instillation method, whereas there is prolonged contact time and increased absorption in the packing method.

Furthermore, Wheatcroft et a ${ }^{13}$ performed a prospective study to compare $5 \mu \mathrm{L}$ microdrops to $25 \mu \mathrm{L}$ drops. There was no statistically significant difference in pupil dilation between the two drop sizes. This result indicates that it is not necessary to use a larger amount of the drug and supports our study. 
There was one case in which the pupil size in the study group was smaller than the control group, and one case that the pupil size was equal in both groups. This was caused by slippage of the soaked cotton wool from the conjunctival fornices. However, we followed the intention-to-treat principle and included them in their respective groups during the analysis.

There were no serious complications such as epithelial defect or keratitis in either group. The most common complication in this study was retained fibers of the cotton wool, which caused mild conjunctivitis. However, in all cases any irritation spontaneously resolved within 1 day after removal.

Regarding cardiovascular side effects, there was no statistically significant difference in mean systolic blood pressure after packing. However, there were statistically significant differences in mean diastolic blood pressure and mean heartbeat after the procedure. The heart rate increased by a statistically significant amount by five beats per minute, which was not clinically significant in any of our cases.

The strength of our findings is underscored by the fact that this is the first comparative study in preterm infants with darkly pigmented irides in a double-blind, controlled, randomized trial. These combine to strengthen the validity of our conclusions. The limitation of this study is in the accuracy of the pupil diameter measurement. Although it would be better to measure with slit-lamp biomicroscope or infrared photography, it is difficult to manage with small preterm infants. However, we measured repeatedly to improve reliability.

In conclusion, we found the lower conjunctival fornix packing of the mydriatric drugs was more effective than the conventional instillation of topical eye drops in mydriasis for preterm infants. The time and the quantity of drugs used are saved, and the safety of the procedure is also acceptable. We recommend the packing method to dilate the pupil of the preterm infants especially in some conditions where the pupils may be difficult to dilate.

Clinical Ophthalmology

\section{Publish your work in this journal}

Clinical Ophthalmology is an international, peer-reviewed journal covering all subspecialties within ophthalmology. Key topics include: Optometry; Visual science; Pharmacology and drug therapy in eye diseases; Basic Sciences; Primary and Secondary eye care; Patient Safety and Quality of Care Improvements. This journal is indexed on Submit your manuscript here: http://www.dovepress.com/clinical-ophthalmology-journal

\section{Acknowledgment}

This study was supported by an invitation research grant (I-50221) from the Faculty of Medicine, Khon Kaen University, Khon Kaen, Thailand.

\section{Disclosure}

The authors report no conflicts of interest in this work.

\section{References}

1. Cryotherapy for Retinopathy of Prematurity Cooperative Group. Multicenter trial of Cryotherapy for retinopathy of prematurity: Snellen visual acuity and structural outcome at $5 \frac{1}{2}$ years after randomization. Arch Ophthalmol. 1996;114(4):417-424.

2. Isenberg SJ, Dang Y, Jotterand V. The pupils of term and preterm infants. Am J Ophthalmol. 1989;108(1):75-79.

3. Sindel BD, Baker MD, Maisels MJ, Weinstein J. A comparison of the pupillary and cardiovascular effects of various mydriatic agents in preterm infants. J Pediatr Ophthalmol Strabismus. 1986;23(6):273-276.

4. Howard HJ, Lee TP. The effect of instillations of ephedrine solution upon the eye. Proc Soc Exp Biol Med. 1927;24:700-702.

5. Yospaiboon Y, Luanratanakorn P, Noppawinyoowong C. Randomized double-blind study of phenylephrine $2.5 \%$ vs $10 \%$ on pupillary dilation. J Med Assoc Thai. 2004;87(11):1380-1384.

6. Angenent WJ, Koelle GB. A possible enzymatic basis for the differential action of mydriatics on light and dark irides. J Physiol. 1953;119(1): 102-117.

7. Emiru VP. Response to mydriatics in the African. Br J Ophthalmol. 1971;55(8):538-543.

8. Khoo BK, Koh A, Cheong P, Ho NK. Combination cyclopentolate and phenylephrine for mydriasis in premature infants with heavily pigmented irides. J Pediatr Ophthalmol Strabismus. 2000;37(1):15-20.

9. Chew C, Rahman RA, Shafie SM, Mohamad Z. Comparison of mydriatic regimens used in screening for retinopathy of prematurity in preterm infants with dark irides. J Pediatr Ophthalmol Strabismus. 2005;42(3):166-173.

10. Ong-Tone L. Use of wick to deliver preoperative mydriatics for cataract surgery. J Cataract Refract Surg. 2003;29(11):2060-2062.

11. McCormick A, Srinivasan S, Harun S, Watts M. Pupil dilation using a pledget sponge: a randomized controlled trial. Clin Experiment Ophthalmol. 2006;34(6):545-549.

12. Dubois V, Wittles N, Lamont M, Madge S, Luck J. Randomised controlled single-blind study of conventional versus depot mydriatic drug delivery prior to cataract surgery. BMC Ophthalmol. 2006;6:36.

13. Wheatcroft S, Sharma A, McAllister J. Reduction in mydriatic drop size in premature infants. Br J Ophthalmol. 1993;77(6):364-365.

\section{Dovepress}

PubMed Central and CAS, and is the official journal of The Society of Clinical Ophthalmology (SCO). The manuscript management system is completely online and includes a very quick and fair peer-review system, which is all easy to use. Visit http://www.dovepress.com/ testimonials.php to read real quotes from published authors. 